\title{
Sensafety: Crowdsourcing the Urban Sense of Safety
}

\author{
Sandro Rodriguez Garzon, Bersant Deva
}

Service-centric Networking, Technische Universität Berlin, Telekom Innovation Laboratories, Berlin, Germany, [sandro.rodriguezgarzon, bersant.deva]@tu-berlin.de

\begin{abstract}
Today, community initiatives to improve the urban quality of life can be conducted in a more focused way because local authorities and urban planners are able to reveal urban hotspots through the investigation of location-annotated crime and accident data. However, urban areas, which according to well-recorded incident data are characterized by a high level of public safety, but which are generally perceived by citizens as unsafe, remain undiscovered and therefore untreated. This work presents Sensafety, a citizen-centric crowdsourcing approach that enables users by means of a mobile application to report their personal feeling of safety anytime and at any site. Sensafety's goal is to reveal a comprehensive and complete picture of the perceived safety in urban environments in order to identify blind spots that have not been further investigated due to lack of data. To encourage citizens to participate and contribute, Sensafety's mobile application offers different ways to explore and experience the collected data depending on the user's location. This paper gives a detailed description of Sensafety's integrated concept and outlines the major technical and non-technical findings.
\end{abstract}

Keywords: crowdsourcing, participatory sensing, public safety, location-based services, geofencing

\section{Introduction}

Studies on the quality of life (QoL) in urban spaces have lately become increasingly popular, especially to compare the QoL of different cities (Mercer, 2019). Most studies are based upon purpose-driven QoL indices that take different combinations of subjective and objective indicators into consideration (McCrea et al., 2006). Objective indicators usually encompass but are not limited to crime statistics, public facilities, public transportation, and state of pollution. Subjective indicators, on the other hand, try to capture the satisfaction and dissatisfaction with the urban living situation through citizen surveys. The fear of crime, as a major subjective indicator and partially determinable by capturing the perceived safety in public spaces, has been proven to have a non-negligible impact on the urban QoL (Michalos and Zumbo, 2000)(Møller, 2005). Another important aspect of the perceived safety is related to the fear to become a victim of an accident. This subjective indicator has so far caught more attention in municipal crowdsourcing campaigns ${ }^{1}$ then in QoL research.

To get a holistic picture of the perceived public safety, consisting of, among others, crime- and traffic-related aspects, within a public space, at least a representative sample of the population needs to be continuously interviewed. Even though this is already practically difficult to achieve, it is aggravated by the fact that perceived safety not only depends on demographics and social factors but also on the location (Kitchen and Williams, 2010) and the situation on site (Doran and Burgess, 2012). In participatory sensing, the required information is not actively requested through cost and time-intensive telephone and street surveys, but is provided voluntarily by the citizens in a proactive way via their personal mobile devices (Burke et al., 2006). Each contribution can thereby be automatically enriched with data originating from smartphone sensors, such as the ge-

\footnotetext{
${ }^{1}$ The Traffic Agent, http://www.trafikkagenten.no
}

ographical location at which the contribution was submitted. Participatory sensing has been successfully applied in related research to capture the personal feeling of crimerelated safety on site (Christin et al., 2013) and the differentiated location-dependent emotions (Hamilton et al., 2011) via mobile applications or the perceived level of danger with respect to traffic via a web (Jiří, 2018) or mobile application (Aubry et al., 2014). The location-annotated data obtained through participatory sensing does not only help to determine the urban QoL in a more differentiated and location-specific manner, but it allows in particular to uncover spots in a city that are perceived to be unsafe with respect to crime or traffic. This knowledge can be used to carry out urban development measures in a more focused way or to increase the presence of law enforcement agencies where and when required.

However, without proper incentives (Restuccia et al., 2016), participatory sensing will not lead to the amount of data required to make meaningful statements about the perceived site-specific safety. In order to encourage volunteers to stay on board and continuously share their personal feeling of safety, the mobile application has to offer free-of-charge value-added services, based on already collected data. This work introduces Sensafety, a citizen-centric mobile application that gives citizens the opportunity to proactively report their personal feeling of safety on site. Sensafety is designed to minimize the required effort for users to share their rating at the expense of the rating's information content. It provides, in addition, multiple value-added services for users to explore and experience the commonly perceived safety in different ways such as via an interactive map or augmented reality view.

The paper starts with a discussion of similar approaches to capture the perceived safety in Section 2. Section 3 introduces Sensafety's integrated concept and describes particular design decisions while Section 4 presents major aspects of the prototypical implementation. The paper fin- 
ishes with concluding remarks and possible use cases in Section 5 and a description of a potential extension and remaining investigations in Section 6.

\section{Related Work}

The urban quality of life with respect to the perception of public safety can be improved, beside many others, by the installation of additional street lights (Painter, 1996) or by an increased presence of law enforcement agencies (Zhao et al., 2002). Where and when to take what kind of action is today decided upon by officials, in most cases, based on data that is already collected for legal reasons, such as for crime or traffic incidents. However, sites that are neither insecure with respect to the local crime rate nor dangerous with respect to the traffic but that are perceived to be unsafe for whatever reason are not considered. In citizen science (Mueller et al., 2012), all citizens of an urban area are encouraged to participate in science projects. Participatory sensing is a concrete variant of it in which humans are considered to act as sensors for geographic information (See et al., 2016) (Berntzen et al., 2018). Due to the omnipresence of mobile devices in everyday life, it is thereby possible to collect location-annotated data on a large scale, area-wide and inexpensively. It is applied in a variety of urban research projects to map urban concerns in general (Ruiz-Correa et al., 2017) or to investigate specific aspects of an urban environment such as accessibility (Prandi et al., 2014), transport network's state (Chen et al., 2013) or infrastructure (Berntzen et al., 2018), or social night life patterns (Santani et al., 2016), to mention just a few.

Crowdsourcing urban human emotions in general or the perceived safety in particular has caught less attention compared to the collection of geo-annotated incidents. Transafe, a mobile platform for crowdsourcing crime and safety perceptions for the city of Melbourne, is a mobile application to report crimes (incidents) but also four different emotions while being in public space (Hamilton et al., 2011). Optionally, comments, pictures or files can be attached to the reports. After aggregation, a map is shown with the aggregated mood at the granularity level of city blocks. The EmoMap mobile application follows a similar path and allows the user to report location-specific emotions via Likert scales and to attach the social context to it (Klettner et al., 2013) (Huang and Gartner, 2016). Instead of providing aggregation results, ratings are published and displayed on a map. The mobile application uSafe allows the user to report the safety feeling via a slider that is annotated with "I am feeling safe", "I am worried" and "I am feeling unsafe" (Christin et al., 2013). In a subsequent step, the user has to choose from eight different crime-related context types such as pickpockets. Reports are aggregated based on a predefined grid and finally being visualized with different granularity levels on a map. uSafe also allows to create retrospective reports for sites the user has visited during the day. The People as Sensors app captures urban emotions as a ground truth for further processing by letting volunteers express their emotions via a mobile application (Resch et al., 2015). For a rating, users select the emotion, e.g. afraid or pleased, the aspect, e.g. safety or traffic, and then submit it after verifying the correctness of the entries.

While Transafe and uSafe put their focus on crime-related perceived safety, the People as Sensors app is more flexible and lets users select from different safety aspects.
EmoMap puts the rating in its social context since the rating level can depend upon whether the safety was perceived while being alone or in company. But EmoMap falls short when it comes to privacy because single ratings are published and become visible to all users. Despite the flexibility of EmoMap and People as Sensors app to address different aspects of perceived safety, they lack required incentives, besides a map for single ratings in EmoMap or a city emotions map immediately after a rating submission within the People as Sensors app, to attract users beyond intrinsically motived volunteers. Transafe and uSafe, on the other hand, offer an always-accessible map view for aggregated results, plus a list view in Transafe. However, it's not clear how Transafe aggregates the emotions on a city block level. uSafe provides, in addition, an alert service based on the ratings, but - similarly - it remains unclear how it determines areas that are rated to be dangerous and how it monitors the user's location with respect to the marked areas in a reliable and energy efficient way.

\section{Concept}

This paper proposes a new integrated concept to leverage the power of crowdsourcing to uncover hotspots in an urban environment that are commonly perceived to be particularly safe or unsafe. The Sensafety concept comprises the citizen-centric mobile application Sensafety (App) and a Sensafety backend (Server). Equipped with the App, citizens are able to proactively and pseudonymously submit their personal site-specific feeling of safety to the Server. The ratings of all users are then centrally processed and the aggregated results being provided back to the App. Location-based services (LBS) within the App are used to present and experience the results in different forms by taking the position of the user into consideration.

\subsection{Rating}

Since the perceived public safety is made up of multiple aspects such as the fear of crime, fear to get involved into a traffic accident or fear to get lost, to mention just a few, it is required for a participatory sensing application to either give the user the opportunity to express a differentiated opinion or to focus only on a single aspect of the public safety. However, the possibility to capture different aspects of the perceived public safety with the help of a single mobile application inevitably leads to a higher cognitive effort for the user, an increased amount of interaction steps and, as a consequence, a longer capturing process. If each user is supposed to capture the aspects of the perceived public safety only once, this is a reasonable approach. If a user should be encouraged to voluntarily share the perceived safety at multiple sites and at different times of the day in order to get a holistic picture of perceived safety across a larger urban environment, this approach is likely to attract lesser long-term motivated users. Capturing only a single aspect of the perceived safety simplifies the user interaction and minimizes the required steps for the user to share the perceived safety. But this goes along with the drawback that the site-dependent perceived safety is not captured in its full complexity. Sensafety's concept follows a different path than the aforementioned approaches and provides users via the App a means to report their general feeling of safety for a site, without specifying the considered aspects (required in uSafe and People as Sensors app) nor its intensities (required in EmoMap, uSafe and Transafe). This 
is intended to simplify and speed up the rating procedure in order to increase the chance that users stay engaged. To keep it even simpler and to ease the on-boarding process, there is no need for users to register (required in EmoMap, Transafe and uSafe) nor the possibility to enter any additional user information, such as age or gender. Although the data collected by Sensafety does not contain any demographics nor contextual indicators for the commonly perceived safety at a site, it reveals out of a macroscopic perspective which sites should be investigated more closely. Sensafety does therefore not intend to replace quantitative and qualitative street interviews but to pinpoint sites that require further investigation.

The perceived safety at a location is subject to constant change. Even slight changes of the environment, e.g. replacement of a broken street lamp, might have a not negligible impact on the perceived safety. Hence, Sensafety allows participants to assess and rate the perceived safety more than once for a given site. With the possibility to rate multiple times, there comes also the danger that users make several deliberately incorrect assessments at a site in order to either encourage officials to take actions although the site is commonly perceived to be safe or to upvalue places that are commonly perceived to be unsafe. Even though the concept of Sensafety is not capable to prevent this fraudulent practice without losing the inherent reevaluation feature, it makes this practice at least more difficult by allowing users to report the perceived safety only once in a given period. Since the perceived safety may significantly vary between day and night, users are allowed to rate the perceived safety at a site twice a day, once during daylight and once at night. Sites are defined as same-sized, predefined, rectangular and non-overlapping geographic areas that are not bound to any administrative entities such as counties or districts (further details on Sensafety's concept of a site are given in Subsection 4.2). If a user already rated for a site, then the user has to either wait for the sunset or sunrise to rate again at the same site or to move and rate at another site. In order to prohibit remote ratings without a proper situational assessment, users are only allowed to rate on site. This design decision prevents users from making any decisions based on hearsay or speculation. It also prevents potentially biased ratings based on human memory, as it is possible in uSafe through retrospective assessments. Once a rating has been submitted, it cannot be withdrawn. This restriction is intended to prevent results from being temporarily falsified by targeted rating and subsequent withdrawal of ratings. The implications on the compliance with the European General Data Protection Regulation (GDPR) are further discussed in Section 4.5.

\subsection{Metrics}

Defining a site as a geographic area rather than a geographical point makes it possible to determine a site-specific metric for the perceived safety, denoted as the Sensafety Index. As a single key value, the Sensafety Index is meant to represent the commonly perceived safety at a site, across the ratings of all participants. The ratings, as opposed to all the aforementioned approaches, are distinguished by the local time of submission, leading to three site-specific outcomes of the Sensafety Index: one for the day, one for the night and another overall assessment. A rating is declared to be a day rating if the local time of submission lies between sunrise and sunset or otherwise, a night rating. De- pending on these time ranges, variants of the subsequent Sensafety Index $I_{n+1}$ for each site are determined through exponential smoothing given by

$$
I_{n+1}=\alpha \cdot r_{n e w}+(1-\alpha) \cdot I_{n}
$$

where $r_{n e w}$ is a numerical representation of a new rating for a site, $I_{n}$ the current Sensafety Index and $\alpha$ the smoothing factor. As opposed to a simple average calculation in uSafe, more recent ratings are considered in Sensafety with a higher weight than older ratings to better reflect the current situation. Since the Sensafety Index is a product of aggregation, it can finally be shared with all users without revealing a single user rating, as opposed to EmoMap where single ratings are published. This property of Sensafety is crucial when it comes to privacy because even though users rate anonymously without providing any demographics, in some cases, the exact position at which a rating was made can be traced back to a certain group of people who, for example, have exclusive access to the site.

\subsection{Incentives}

Besides the goal to provide a simple and fast way to report the perceived safety, the App needs to offer additional incentives in order to foster engaged citizens, with an intrinsic motivation to improve their neighborhood, to rate on a regular basis and to encourage less active citizens to start contributing. For that purpose, the App offers buildin value-added LBS's that let users explore and experience the aggregated results from a global or local point of view. The global view is provided by visualizing the different Sensafety Indices for all sites on an interactive map, similar to the approach taken by uSafe. Users are then able to examine the Sensafety Index for all sites for which at least one rating was submitted. A local view onto the results is provided by an augmented reality (AR) view and a safety compass. Both views take the current position of the user into consideration to visualize Sensafety Index information from within the vicinity of the user. In the AR view, the camera view is annotated with Sensafety Index information while the safety compass indicates how the safety is commonly perceived in all cardinal directions, with the reference position given by the user's location. While the global and local views are intended to be used for exploration purposes only, an alert service is provided in addition to experience the hyper-local Sensafety Indices while on the go. It proactively alerts the user when she/he is about to enter a hot spot where the average level of perceived safety falls below a user-defined threshold.

\section{Implementation}

The App and Server are the key components of the Sensafety concept. The App contains all user-facing elements and is implemented for both leading mobile operating systems Android and iOS in order to ensure the availability for all citizens with access to these mobile ecosystems. Its design and features has been developed in close collaboration with potential users. Sensafety's server-sided implementation enables the aggregated computation of the Sensafety Index and its provision to the App as a common knowledge base. This section provides a more detailed view on the implementation of Sensafety's core aspects, including the realization of the rating view, the computation of the 


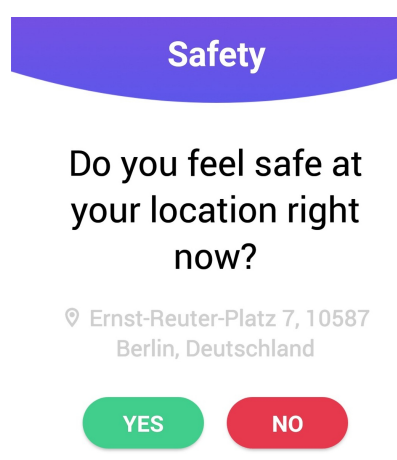

Figure 1. Sensafety's rating view with the dichotomous question on perceived safety

Sensafety Index and its aggregation, as well as the presentation of featured incentives, such as the compass and AR view and the adaptive alerts. It concludes with a discussion on measures taken to protect the user's privacy and to ease the App's use within the sensitive topic of perceived safety.

\subsection{Rating View}

The App uses a distinct view for the rating of the perceived safety. It is quickly accessible throughout the App via a centrally positioned button. The rating view (shown in Figure 1) contains the single dichotomous question "Do you feel safe at your location right now?", which has been carefully constructed to encapsulate context-sensitive aspects. Its wording contains the spatial (at your location) and temporal (right now) context information in which the question is expected to be answered. In addition to the time, which is visible on the status bar of mobile devices, the human-readable address of the current location is shown to make the user aware to which site the rating is accounted for before it is submitted. Since the question is quite complex, the answer option is kept binary with colored "YES" and "NO" buttons. A binary is favored over a gradual selection (uSafe, EmoMap and Transafe use gradual selection), because it reduces the mental effort to answer the question on the go. The user should immediately give a rating based on gut feeling without worrying about how intense the feeling is and where to locate it on a scale. This type of rating is intended to encourage users to quickly express their safety feeling in different situations, places and times during the day. Potential situations should explicitly include circumstances in which quick responding is essential, e.g. while passing through crime-affected neighborhoods. Sensafety requires explicit assessments by the user, so that the question is only answered if the user is willing and has no ambiguities in his or her safety feelings for the site. If the user is in doubt, the rating process can be canceled easily at any time. As emphasized before, the rating view for a specific site can only be accessed and answered when the user is located on that site and only twice per day during daylight and night. Once the question has been answered by a user, the rating is sent to the Server and contains a hashed pseudonomous identifier, a local timestamp, a daylight flag, the geographical location, the site identifier, and the answer.

\subsection{Sensafety Index Computation}

The Sensafety Index for a site is computed on the Server based on ratings as presented in equation 1 . Sensafety

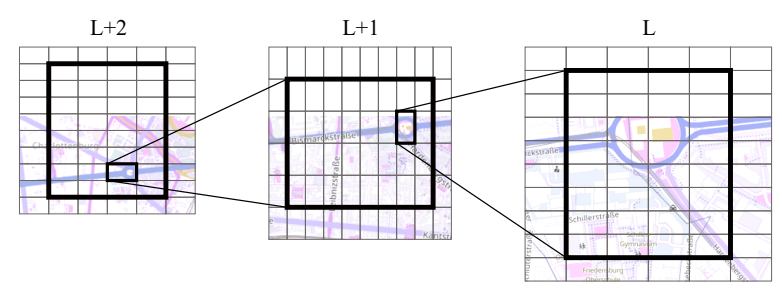

Figure 2. Grid-based aggregation of the Sensafety Index for different levels of precision at different zoom levels

uses the spatial, hierarchical and rectangular geohash (Niemeyer, 2008) data structure for the representation of sites as grid cells. Figure 3 a shows the interactive map with the calculated index at different sites alongside UI elements to show the number of collected ratings for the view area and a switch to change between day, night and overall Sensafety Indices. Users are able to immediately grasp the perceived safety of their city through the colorization of sites from green to red. By pressing on a rated site, it reveals a colored gauge with the corresponding Sensafety Index and the number of ratings. As mentioned above, Sensafety favors early and more recent ratings. The smoothing factor $\alpha$ is dependent on the number of ratings $n$ for a particular site as shown in Table 1 . This ensures that early ratings at a site have a higher impact on the overall result of the index, encouraging users to express their perception of safety at an unrated site. With a significant number of ratings $(\geq 100)$, the smoothing factor stabilizes at a value of 0.2 and thus continues to emphasize the most recent rating with $20 \%$ of the overall index.

\begin{tabular}{|c||c|c|c|c|c|c|c|c|c|}
\hline $\mathrm{n}$ & 0 & $<5$ & $<10$ & $<25$ & $<40$ & $<60$ & $<80$ & $<100$ & $\geq 100$ \\
\hline$\alpha$ & 1 & 0.7 & 0.5 & 0.4 & 0.35 & 0.3 & 0.25 & 0.22 & 0.2 \\
\hline
\end{tabular}

Table 1. Exponential smoothing $\alpha$ parameters depending on the number of ratings $n$ per site

Geohashes use the Z-order curve and a variant of base 32 encoding for its computation, thus, each geohash (representing a grid cell) consists of 32 sub-cells with a higher precision at every lower hierarchical level. The Sensafety Index is initially calculated for the precision of geohash length 7. A site is therefore defined in Sensafety to be a grid cell with a dimension of around $152.9 \mathrm{~m} \mathrm{x} 152.4 \mathrm{~m}$ at the equator and is uniquely identified by a geohash of length 7. This extent of a site has been carefully selected because of two reasons. Due to the anonymous nature of Sensafety and the sensitivity of information, grid cell dimensions should be large enough so that it is unlikely that ratings can be attributed to individual users, however, at the same time small enough so that grid cells depict the actual size of an urban site properly. The interactive map adapts the visualization of the rated sites based on the zoom level. When zoomed out, the map shows an aggregated level of the Sensafety Index using higher level grid cells with a geohash of shorter length (see Figure 2). The aggregation of higher level grid cells is computed by the weighted sum

$$
I_{L+1}=\sum_{i=1}^{32} I_{L[i]} \cdot w_{L[i]}
$$

where $I_{L+1}$ is the aggregated Sensafety Index for a higher level grid cell, $I_{L[i]}$ is the Sensafety Index of the $i$-th sub- 


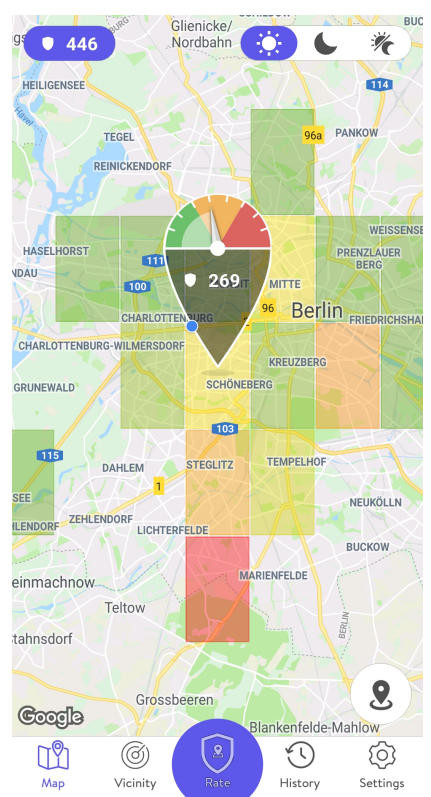

(a) Map View

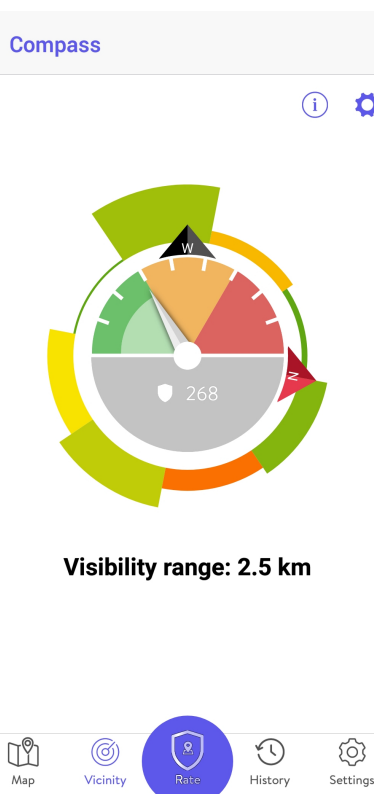

(b) Compass View

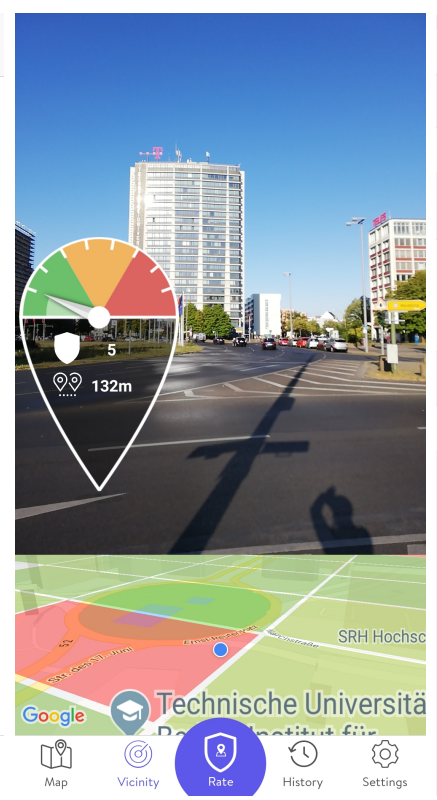

(c) AR View

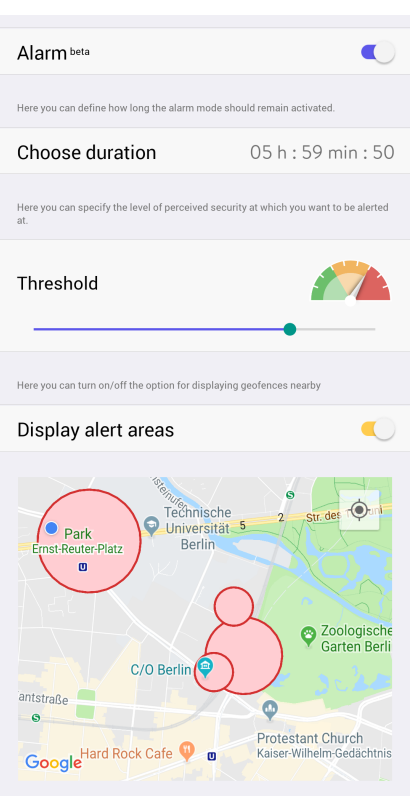

(d) Alert Service View

Figure 3. Sensafety App Views

cell, and $w_{L[i]}$ is the weight of the $i$-th sub-cell calculated by the number of ratings in the sub-cell divided by the total number of ratings on the higher level $L+1$. It should be noted that unrated sites at the lowest level 7 are not considered for the aggregation as the weight for these cells will be 0 .

\subsection{Vicinity Views}

Sensafety uses hyper-local views to let the users investigate the perceived safety information within their vicinity. This is accomplished by using modern means of context-aware visualizations and interactions within a compass (see Figure $3 b$ ) view and an AR (see Figure 3c) view.

Compass View: The safety compass view provides an immediate overview on the perceived safety by aligning the Sensafety Index with the cardinal directions. It depicts the previously used colored gauge containing the Sensafety Index and the number of ratings for the site the user is currently located in at the center of the screen. Indicators of the northern and current cardinal direction the device is facing towards are placed to provide users with direct orientation. The varying sized areas at the verge of the compass show aggregated ratings at eight directions of surrounding sites within a user-defined visibility range. This verged area computation is based on the compass sensor of the mobile devices and on angle-, distance- and numberof-ratings-based weighted averages. For this, the distance and angle between the user's location and the centers of encompassing sites within the visibility range are calculated. Closer distanced sites with higher number of ratings and angles pointing closer to the eight directions are weighted linearly higher for the aggregated rating. Sizes of the verged areas are determined by the number of ratings, thus, the larger the areas are, the more ratings were given in the respective direction providing users with a measure of confidence in the rating. For example, a large red area in a certain direction would indicate a high number of ratings with a low perceived safety in that direction.
Augmented Reality View: The AR view allows users to experience the location-specific insights using the built-in camera. It dynamically overlays Sensafety's colored gauge (displaying Sensafety Index and amount or ratings) on the real world camera view based on the position, the tilt and direction of the mobile device. By looking down through the camera, insights for the currently visited site are displayed while looking at the horizon reveals the insights of directly neighboring sites. In order to retrieve a rotation matrix $R$ of the horizontal and vertical orientation of the device the gyroscope sensor is used. The AR view uses systematic matrix transformations through several coordinate systems to be able to overlay the markers of the sites on the mobile device's screen. At first, a world space matrix $W(i)$, depicting the relative coordinate space between the $i$-th sites on the lowest level 7 and the user is computed, by converting the closest sites to Mercator coordinates and subtracting each by the user's geographic location. Additionally, a screen space matrix $S$ and perspective space matrix $P$ are introduced to adapt to the screen size and format as well as the perspective of projected entities on the screen. The resulting 4D matrix transformation is computed as follows:

$$
A R(i)=S \times P \times R \times W(i)
$$

To extract the point coordinates for the AR view, a 2D projection is used to position the gauge elements on the user's screen. The AR view also contains a tilted map in the lower third of the screen using the rotation matrix to pan alongside the user's movement. This screen introduces an enhanced experience and gives the user orientation and information from two different perspectives.

\subsection{Adaptive Alerts}

While the App maintains a holistic set of views for exploring the Sensafety Index in the vicinity of users, chances are that areas perceived as unsafe might be missed when the 


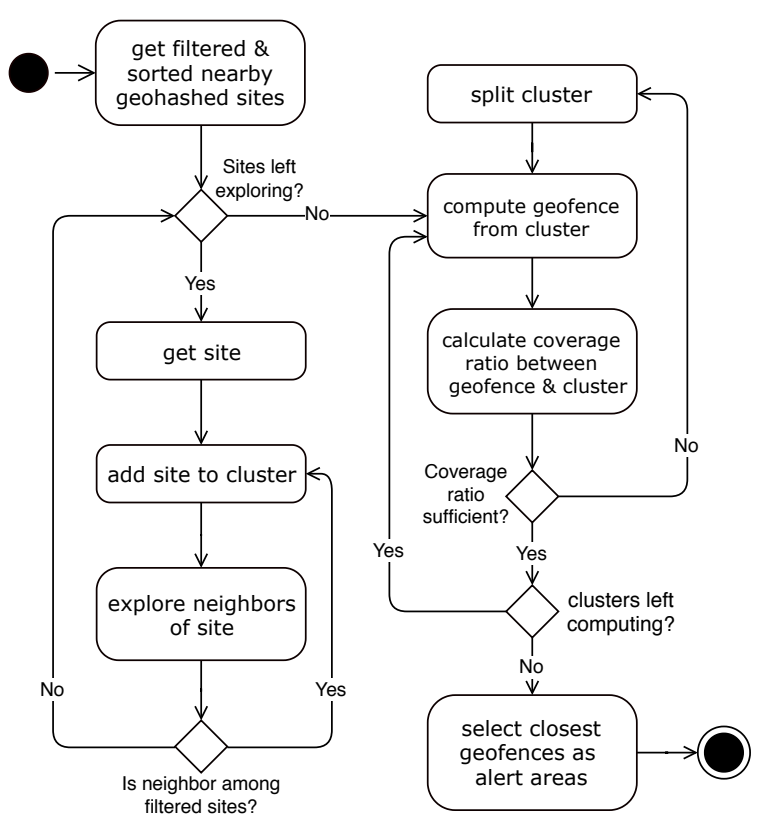

Figure 4. Activity diagram depicting the alert area computation process from sites to geofences.

App is not actively used. For this reason, adaptive alerts are introduced that enable users to get proactively notified as they approach areas that are perceived to be unsafe. Figure $3 \mathrm{~d}$ shows the In-App interaction screen in the notifications settings. Users can enable or disable the alerts, as well as choose a duration for its operation. A sensitivity threshold can be set by the familiar colored gauge that is being used throughout the various means of visualization. Once the alert function has been activated, the computed alert areas are shown on a map view below. The continuous monitoring of these alert areas is carried out via the geofencing APIs, which are specific to the respective mobile operating system and vendor. Geofencing (Küpper et al., 2011) describes the process by which events are proactively triggered when a geographically defined area (geofence) is entered or left. To provide reliable and battery efficient geofencing, vendors' APIs allow only circular shapes and a limited number of simultaneously monitored geofences. These limitations need to be taken into consideration for the computation process of the alert areas, which is depicted in Figure 4.

In an initial step, a set of sites that surpassed the userdefined threshold in the vicinity of the user's location are queried. This filtered set of nearby sites is sorted by the current distance between each site and the user's location, thus limiting the computation to relevant close-by sites only. Based on this set, cohesive clusters of areas that are perceived to be unsafe are computed. For this purpose, the site set is iteratively explored and expanded towards their neighbors to form common clusters. If neighbors of a site are among the nearby filtered site set, they are integrated into a common cluster and further expanded upon. Once all sites have been explored and the initial set of clusters has been formed, geofences for each cluster are computed. Each geofence consists of a point and a radius. The point is calculated as the centroid of all sites within a cluster, whereas the radius is taken as the maximum distance between all elements of a cluster and its centroid. This approach makes sure that all sites perceived to be unsafe are

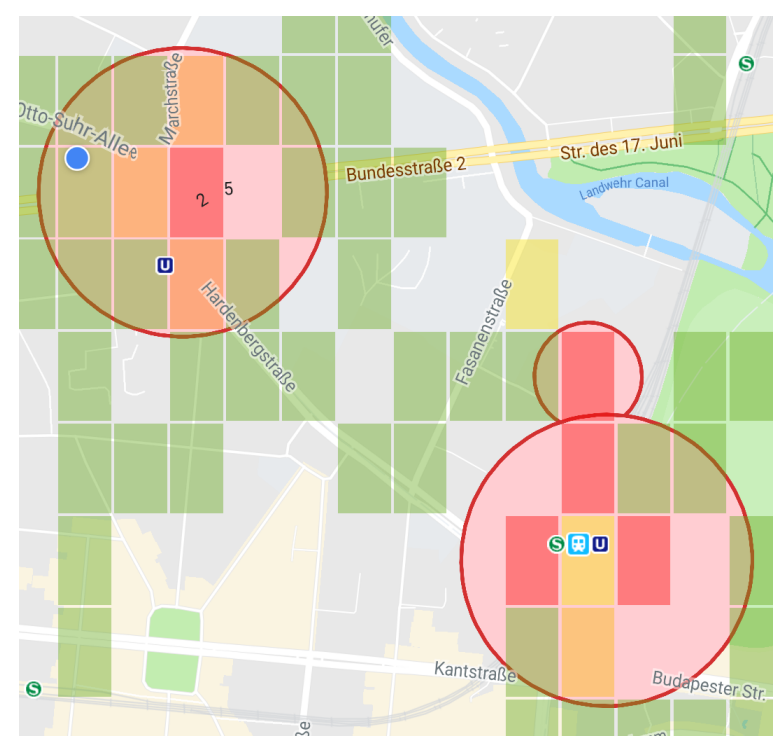

Figure 5. Computed alert areas surrounding sites perceived to be unsafe in the area of Berlin-Charlottenburg

covered by a geofence, however, it also leads to large sized alert areas when cluster elements are mostly at the edge of the cluster, for example, in long consecutive chains. In order to reduce the size of these large geofences, the coverage ratio between the surfaces of the clustered sites and the computed geofence is calculated. In case the coverage ratio is not sufficient the cluster is split into multiple ones. The selection process for the split is determined by finding sites within the cluster that have the most neighboring sites. These sites are then selected together with their neighbors as the new split cluster. After all clusters have been computed to geofences and the sufficient coverage ratio has been reached, the geofences closest to the user are selected as alert areas to be monitored. Figure 5 depicts the resulting alert areas in circular shape surrounding the clusters of sites perceived to be unsafe.

The computation of alert areas takes place not only when the alert service is activated through the user interface, but also when certain contextual conditions are met in order to keep the state of the indices and the alert areas around the user up-to-date at all times. As illustrated above, the alert area computation process is strongly based on Sensafety's underlying dynamic data model and only considers the closest areas to the user. Therefore, to stay relevant for users with the most recent alert areas, the service reinitiates the computation process with current parameters, and thus adapts the alert areas to three different kind of contextual changes. First, Sensafety triggers recalculations when users move to other locations that are beyond the range of the currently computed set of alert areas. Second, the Sensafety Index updates itself periodically for nearby sites when alerts are activated, potentially leading to the recalculation of alert areas by updating the Sensafety Indices. Finally, alerts adapt to day- and night-time ratings and follow sunrise and sunset intervals. The computations and the continuous background tracking used by the mobile operating systems to provide the geofencing functions are exclusively executed on the users' mobile devices. The alerts appear as notifications on the respective mobile operating systems when entering the alert area and disappear once the area has been left. 


\subsection{Privacy}

Once the App is installed, the user can start to rate immediately, without the need to register with personal credentials. However, the significant simplified onboarding process makes it difficult for Sensafety to prevent malicious users bypassing the restriction to rate at most twice per day for a single site. A user might, for example, try to uninstall and re-install the mobile application to reset its state. Assuming ratings are anonymous, Sensafety would then not be able to detect after a reinstall whether a user has already rated a particular site before. So instead of introducing non-anonymous ratings by means of a mandatory user registration, Sensafety makes use of unique pseudonomous identifiers for mobile devices that remain the same even after resetting or reinstalling the mobile application. Since each submitted rating contains a hashed version of the pseudonomous identifier, Sensafety is able to detect and discard maliciously submitted ratings. Even though the hashed pseudonomous identifier within a rating does not reveal the identity of the user nor the device type, it can be used to track a device across multiple ratings in order to infer the user's personal identity (Krumm, 2007). Although the user is not able to withdraw a rating as outlined above, she/he is offered the opportunity to request a full anonymization of all past user ratings given the pseudonomous identifier. This complies with a required data deletion policy as defined by the European GDPR since the personal information stored by Sensafety, the hashed version of the pseudonomous identifier, can be removed on demand. Malicious use is therefore still possible, but the process to bypass the restrictions requires more time and effort. The Sensafety Index computation and its aggregation complies with the GDPR as no personal data in form of the hashed pseudonomous identifier is attached to these site-specific indices.

Despite this privacy-preserving property of the Sensafety Index, it still remains, as indicated above, the possibility to trace back all ratings within a rectangular geohash-based area of about $152.9 \mathrm{~m} \mathrm{x} 152.4 \mathrm{~m}$ (at equator) in case that area lies entirely within a greater area where only a limited set of people have access to. While this scenario is not a big issue for dense urban areas, it is fairly probable in rural areas. It must be emphasized that Sensafety is aiming to determine the perceived safety in public spaces and not within private properties. Sensafety has no build-in support to detect whether a rating has been submitted for a public space or a private property. All submitted ratings are treated the same way. Therefore, it is the responsibility of the user to not submit ratings for private spaces. These ratings would be, anyhow, of no interest to public authorities or urban planners.

\section{Conclusion}

This work presented an integrated approach to capture personal site-specific perceived safeties within urban environments through the citizen's mobile devices. Since citizens are supposed to proactively report their personal perceived safety voluntarily and more than once by means of a mobile application, a simple rating interaction concept with a single dichotomous question was favored over capturing all potential aspects of the perceived safety, their intensities and weighting via a more complex and time-consuming user interface. Although the captured data does not allow conclusions to be drawn about the reasons behind a particular rating, it does help to narrow down the search for unknown hotspots within a city that are, with respect to the perceived safety, worth to be investigated in more detail by traditional means. The visualization of site- and timespecific Sensafety Indices by means of an interactive map, augmented reality view and a safety compass, and a corresponding alert service should not only make the common urban sense of safety accessible to users but it should also encourage them to contribute on a regular basis.

However, Sensafety's main task remains to quantify the general urban feeling of safety, without the need to rely on word-of-mouth recommendations. But it remains to be seen, whether Sensafety's integrated concept and its concrete implementation is able mobilize a critical mass of contributers so as to provide a sufficient and sound data basis for further investigations. The results might be used in many ways to improve, facilitate or understand life in urban areas. Depending on the point of view, residents can take Sensafety as a means to participate in urban planning processes, visitors can inquire Sensafety about the situation on site, law enforcement agencies can identify hidden hotspots, politicians can address local specificities in their campaigns, realtors can consider the Sensafety Indices during the estimation of real estate prices, insurances can offer more differentiated rates and scientists can make use of the data to investigate the factors that influence the perceived urban safety. This is only a small selection of the wide range of potential applications and by no means a complete list.

\section{Future Work}

From the conceptional point of view, the proposed approach can be extended with the possibility for Sensafety's mobile application to automatically initiate an interview with the user based on the user's location, rating history and commonly perceived safety at a site. This applicationinitiated interview can then be used to learn more about the user's attitude, without the need to extend the simplified user-initiated rating process. Such an unsolicited inquiry by Sensafety should, however, be carefully selected and only take place when the user enters a site under investigation for which the personal ratings show a clear tendency that match with the commonly perceived safety at a site. For example, instead of proactively alerting the user when entering an alert area, Sensafety could automatically ask the user to name the aspect, e.g. crime or accident, that the user mainly addresses when she/he reports a low perceived safety for that site.

In general, Sensafety's concept is based upon the fundamental assumption that volunteers contribute in a truthful manner. However, if the personal rating shared with Sensafety indirectly leads to a deterioration of the local quality of life, then volunteers may be encouraged to stop rating in a truthful manner. For example, visitors equipped with Sensafety may avoid to visit a neighborhood that is perceived by its residents to be unsafe. This could even contribute to a worsening of the situation on site which may, in turn, trigger residents to report untruthful ratings. Sensafety concept should therefore be further examined as to whether and how such behavior can be detected, whether 
incorrect assessments have a significant influence on the overall perceived safety at a site and how Sensafety can potentially deal with incorrect assessments. It must, in addition, be investigated, whether citizens are willing to rate in alleged dangerous situations and how visitor ratings might bias the overall results for a neighborhood.

\section{Acknowledgments}

We would like to thank Fabian Puch, Michael Raring, Jonathan Zimmermann, Nino Filiu, Victor Morel, Marcel Reppenhagen, Michał Zwolak, Mikołaj Robakowski, Yared D. Dessalk, Martin Kachev and Denis Rangelov for their valuable contribution to the Sensafety project.

\section{References}

Aubry, E., Silverston, T., Lahmadi, A. and Festor, O., 2014. CrowdOut: a Mobile Crowdsourcing Service for Road Safety in Digital Cities. In: 2014 IEEE Int. Conf. on Pervasive Computing and Communication Workshops (PERCOM WORKSHOPS), pp. 86-91.

Berntzen, L., Johannesen, M. R., Böhm, S., Weber, C. and Morales, R., 2018. Citizens as Sensors - Human Sensors as a Smart City Data Source. In: Proc. of the 7th Int. Conf. on Smart Cities, Systems, Devices and Technologies.

Burke, J., Estrin, D., Hansen, M., Parker, A., Ramanathan, N., Reddy, S. and Srivastava, M. B., 2006. Participatory Sensing. In: First Workshop on World-Sensor-Web: Mobile Device Centric Sensory Networks and Applications (WSW'06).

Chen, K., Lu, M., Tan, G. and Wu, J., 2013. CRSM: Crowdsourcing based Road Surface Monitoring. In: 2013 IEEE 10th Int. Conf. on High Performance Computing and Communications \& 2013 IEEE Int. Conf. on Embedded and Ubiquitous Computing, pp. 2151-2158.

Christin, D., Roßkopf, C. and Hollick, M., 2013. "uSafe: A Privacy-aware and Participative Mobile Application for Citizen Safety in Urban Environments". Pervasive and Mobile Computing 9(5), pp. 695 - 707.

Doran, B. J. and Burgess, M. B., 2012. Putting Fear of Crime on the Map: Investigating Perceptions of Crime Using Geographic Information Systems. Springer Series on Evidence-Based Crime Policy, 1 edn, Springer-Verlag New York.

Hamilton, M., Salim, F., Cheng, E. and Choy, S. L., 2011. Transafe: a Crowdsourced Mobile Platform for Crime and Safety Perception Management. In: 2011 IEEE Int. Symposium on Technology and Society (ISTAS), pp. 1-6.

Huang, H. and Gartner, G., 2016. Using mobile crowdsourcing and geotagged social media data to study people's affective responses to environments. European Handbook of Crowdsourced Geographic Information pp. 385-399.

Jiří, P., 2018. Participatory mapping in community participation - case study of Jeseník, Czech Republic. Quaestiones Geographicae 37(3), pp. 151-162.

Kitchen, P. and Williams, A., 2010. Quality of Life and Perceptions of Crime in Saskatoon, Canada. Social Indicators Research 95(1), pp. 33-61.

Klettner, S., Huang, H., Schmidt, M. and Gartner, G., 2013. Crowdsourcing affective responses to space. Kartographische Nachrichten 63, pp. 66-73.

Krumm, J., 2007. Inference Attacks on Location Tracks. In: A. LaMarca, M. Langheinrich and K. N. Truong (eds), Proc. of the 5th Int. Conf. on Pervasive Computing, Pervasive 2007, Springer Berlin Heidelberg, pp. 127-143.
Küpper, A., Bareth, U. and Freese, B., 2011. Geofencing and Background Tracking - The Next Features in LBSs. In: H.-U. Heiß, P. Pepper, H. Schlingloff and J. Schneider (eds), INFORMATIK 2011, Lecture Notes of Informatics, Vol. P192, Gesellschaft für Informatik e.V., Bonn.

McCrea, R., Shyy, T.-K. and Stimson, R., 2006. What is the Strength of the Link Between Objective and Subjective Indicators of Urban Quality of Life? Applied Research in Quality of Life 1(1), pp. 79-96.

Mercer, 2019. Quality of Living Ranking. https: //mobilityexchange.mercer.com/Insights/ quality-of-living-rankings. [Accessed 2019-09$16]$.

Michalos, A. C. and Zumbo, B. D., 2000. Criminal Victimization and the Quality of Life. Social Indicators Research 50(3), pp. 245-295.

Møller, V., 2005. Resilient or Resigned? Criminal Victimisation and Quality of Life in South Africa. Social Indicators Research 72(3), pp. 263-317.

Mueller, M. P., Tippins, D. and Bryan, L., 2012. The Future of Citizen Science. Democracy and Education.

Niemeyer, G., 2008. Geohash. http://geohash.org, Accessed: 2019-09-16.

Painter, K., 1996. The influence of street lighting improvements on crime, fear and pedestrian street use, after dark. Landscape and Urban Planning 35(2), pp. 193 201. Streets Ahead.

Prandi, C., Salomoni, P. and Mirri, S., 2014. mPASS: Integrating People Sensing and Crowdsourcing to Map Urban Accessibility. In: 2014 IEEE 11th Consumer Communications and Networking Conf. (CCNC), pp. 591-595.

Resch, B., Summa, A., Sagl, G., Zeile, P. and Exner, J.-P., 2015. Urban Emotions-Geo-Semantic Emotion Extraction from Technical Sensors, Human Sensors and Crowdsourced Data. Springer International Publishing, Cham, pp. 199-212.

Restuccia, F., Das, S. K. and Payton, J., 2016. Incentive Mechanisms for Participatory Sensing: Survey and Research Challenges. ACM Transactions on Sensor Networks 12(2), pp. 13:1-13:40.

Ruiz-Correa, S., Santani, D., Ramírez-Salazar, B., Ruiz-Correa, I., Rendón-Huerta, F. A., Olmos-Carrillo, C., Sandoval-Mexicano, B. C., Arcos-García, A. H., Hasimoto-Beltrán, R. and Gatica-Perez, D., 2017. SenseCityVity: Mobile Crowdsourcing, Urban Awareness, and Collective Action in Mexico. IEEE Pervasive Computing 16(2), pp. 44-53.

Santani, D., Biel, J.-I., Labhart, F., Truong, J., Landolt, S., Kuntsche, E. and Gatica-Perez, D., 2016. The Night is Young: Urban Crowdsourcing of Nightlife Patterns. In: Proc. of the 2016 ACM Int. Joint Conf. on Pervasive and Ubiquitous Computing, UbiComp '16, ACM, New York, NY, USA, pp. 427-438.

See, L., Mooney, P., M. Foody, G., Bastin, L., Comber, A., Estima, J., Fritz, S., Kerle, N., Jiang, B., Laakso, M., Liu, H.-Y., Milčinski, G., Nikšič, M., Painho, M., Pődör, A., Olteanu Raimond, A.-M. and Rutzinger, M., 2016. Crowdsourcing, Citizen Science or Volunteered Geographic Information? The Current State of Crowdsourced Geographic Information. ISPRS Int. Journal of Geo-Information.

Zhao, J. S., Schneider, M. and Thurman, Q., 2002. The effect of police presence on public fear reduction and satisfaction: A review of the literature. The Justice Professional 15(3), pp. 273-299. 